\title{
CORRECTION
}

\section{Correction to: Study of Cellular Aging in a Cohort of Patients with Heart Failure}

\author{
Elena Bularca ${ }^{1}$ - María Izarbe Merino-Casallo ${ }^{2,3}$ · Susana Olivera-González ${ }^{1,2,3}$. Sebastián Menao-Guillén ${ }^{1,2,4}$. \\ José Luis Sierra-Monzón ${ }^{2,3}$. José María Domingo-Morera ${ }^{2,5} \cdot$ Miguel Ángel Torralba-Cabeza $^{1,2,3}$ (1)
}

Published online: 11 May 2021

(c) Italian Society of Hypertension 2021

\section{Correction to: \\ High Blood Pressure \& Cardiovascular Prevention (2021) 28:49-55 \\ https://doi.org/10.1007/s40292-020-00421-x}

\section{Author names that read as:}

Bularca Elena, Merino-Casallo María Izarbe, OliveraGonzález Susana, Menao-Guillén Sebastián, Sierra-Monzón José Luis, Domingo-Morera José María, Torralba-Cabeza Miguel Ángel

\section{Should read}

Elena Bularca, María Izarbe Merino-Casallo, Susana Olivera-González, Sebastián Menao-Guillén, José Luis SierraMonzón, José María Domingo-Morera, Miguel Ángel Torralba-Cabeza

The original article can be found online at https://doi.org/10.1007/ s40292-020-00421-x.

Miguel Ángel Torralba-Cabeza

mantorralba@gmail.com

1 School of Medicine, University of Zaragoza, Domingo Miral Street S/N, 50009 Zaragoza, Spain

2 Aragon Health Research Institute (IIS Aragon), San JuanBosco Avenue, 50009 Zaragoza, Spain

3 Department of Internal Medicine, "Lozano Blesa" UniversityHospital, 15th, San Juan Bosco Avenue, 50009 Zaragoza, Spain

4 Department of Biochemistry, "Lozano Blesa" UniversityHospital, 15th, San Juan Bosco Avenue, 50009 Zaragoza, Spain

5 Bank of Blood and Tissues of Aragon, Medical Department, 1st, Ramón Salanova Street, 50017 Zaragoza, Spain 